established by an information letter, personally addressing each potential participant. To increase the response rate a lottery incentive was given. At-risk criteria for psychosis were assessed with the Schizophrenia Prediction Instrument, Adult version (SPIA) and the Structured Interview for Prodromal Syndromes (SIPS). Results: $4.9 \%$ of the sample $(n=5)$ turned out as ineligible. Of the remaining 97 persons (eligibility fraction: 95.1\%), sufficient contact details, i.e. address plus telephone number, could be identified for 85 persons (enrolment fraction: 87.6\%). Of the enrolment fraction, 60 participated (recruitment fraction: 70.6\%). Of the 21 non-responders, 6 (7.1\%) gave current lack of time for reason for non-participation but voiced general willingness to participate within 1-6 months time; only 15 (17.6\%) definitely refused to participate. $4(4.7 \%)$ could not be reached or were still indecisive about participation at conclusion of the pilot. Most of the definite refusers reasoned with lack of interest in the study. While no differences between participants and refusers were detected regarding gender, community, educational and occupational level, an age effect was evident: The youngest age group (16-20 years), at a participation rate of $58.2 \%$, was least likely to participate. All participants rated the questions as pleasant, experienced no burden by the interview and agreed to be re-contacted.

Discussion: A population survey on at-risk criteria for psychosis in the Canton Bern seems feasible. The interviews were well tolerated. The information letter and the lottery turned out to be important in achieving the targeted participation rate. Yet, one recruitment problem needs to be considered: The lower participation rate amongst the younger participants. Measures to increase participation rate in this age group are necessary, i.e. "call for participation" in a widely read newspaper or a study webpage with additional Background information to raise interest in the study.

doi:10.1016/j.schres.2010.02.270

\section{Poster 43 \\ METABOLIC ALTERATIONS IN THE CORTEX OF A MOUSE MODEL WITH GLUTATHIONE DEFICIT - RELEVANCE TO SCHIZOPHRENIA}

Joao M.N. Duarte ${ }^{1}$, Anita Kulak ${ }^{2}$, Rolf Gruetter ${ }^{1,3}$, Kim Q. Do ${ }^{2}$

${ }^{1}$ Laboratory for functional and metabolic imaging, Center for Biomedical Imaging, Ecole Polytechnique Federale Lausanne, Lausanne, Vaud, Switzerland; ${ }^{2}$ Centre for Psychiatric Neuroscience, Lausanne University Hospital, Lausanne, Vaud, Switzerland; ${ }^{3}$ Department of Radiology, Universities of Lausanne and Geneva, Geneva and Lausanne, Geneva/ Vaud, Switzerland

Background: Glutathione (GSH) is a major redox regulator and antioxidant and is decreased in cerebrospinal fluid and prefrontal cortex of schizophrenia patients [Do et al. (2000) Eur J Neurosci 12:3721]. The genes of the key GSH-synthesizing enzyme, glutamate-cysteine ligase catalytic (GCLC) and modifier (GCLM) subunits, are associated with schizophrenia, suggesting that the deficit in GSH synthesis is of genetic origin [Gysin et al. (2007) PNAS 104:16621]. GCLM knock-out (KO) mice, which display an $80 \%$ decrease in brain GSH levels, have abnormal brain morphology and function [Do et al. (2009) Curr Opin Neurobiol 19:220]. Developmental redox deregulation by impaired GSH synthesis and environmental risk factors generating oxidative stress may have a central role in schizophrenia. Here, we used GCLM KO mice to investigate the impact of a genetically dysregulated redox system on the neurochemical profile of the developing brain.

Methods: The neurochemical profile of the anterior and posterior cortical areas of male and female GCLM KO and wild-type mice was determined by in vivo ${ }^{1} \mathrm{H}$ NMR spectroscopy on postnatal days $10,20,30,60$ and 90 , under 1 to $1.5 \%$ isoflurane anaesthesia.
Localised ${ }^{1} \mathrm{H}$ NMR spectroscopy was performed on a $14.1 \mathrm{~T}, 26 \mathrm{~cm}$ VNMRS spectrometer (Varian, Magnex) using a home-built $8 \mathrm{~mm}$ diameter quadrature surface coil (used both for RF excitation and signal reception). Spectra were acquired using SPECIAL with TE of $2.8 \mathrm{~ms}$ and TR of $4 \mathrm{~s}$ from VOIs placed in anterior or posterior regions of the cortex [Mlynárik et al. (2006) MRM 56:965]. LCModel analysis allowed in vivo quantification of a neurochemical profile composed of 18 metabolites.

Results: GCLM KO mice displayed nearly undetectable GSH levels as compared to WT mice, demonstrating their drastic redox deregulation. Depletion of GSH triggered alteration of metabolites related to its synthesis, namely increase of glycine and glutamate levels during development (P20 and P30). Concentrations of glutamine and aspartate that are produced from glutamate were also increased in GCLM KO animals relative to WT. In addition, GCLM KO mice also showed higher levels of $N$-acetylaspartate that originates from the acetylation of aspartate. These metabolites are particularly implicated in neurotransmission processes and in mitochondrial oxidative metabolism. Their increase may indicate impaired mitochondrial metabolism with concomitant accumulation of lactate in the adult mice (P60 and P90). In addition, the GSH depletion triggers reduction of GABA concentration in anterior cortex of the P60 mice, which is in accordance with known impairment of GABAergic interneurons in that area. Changes were generally more pronounced in males than in females at P60, which is consistent with earlier disease onset in male patients.

Discussion: In conclusion, the observed metabolic alterations in the cortex of a mouse model of redox deregulation suggest impaired mitochondrial metabolism and altered neurotransmission. The results also highlight the age between $\mathrm{P} 20$ and P30 as a sensitive period during the development for these alterations.

doi:10.1016/j.schres.2010.02.271

\section{Poster 44 \\ THE DIFFERENCES OF 2ND TO 4TH DIGIT LENGTH RATIO BETWEEN SCHIZOPHRENIA PATIENTS AND NORMAL CONTROLS}

\author{
Yu Sang Lee ${ }^{1}$, Hyun-Woo Cho ${ }^{1}$, Jeong-Ho Chae ${ }^{2}$ \\ ${ }^{1}$ Yongin Mental Hospital, Yongin, Gyeonggi, South Korea; ${ }^{2}$ Catholic \\ University, College of Medicine, Seoul, South Korea
}

Background: The ratio of 2nd to 4th finger(2D:4D) is known to be an indirect measure for prenatal sex hormone exposure. Sex hormone influences the brain development through structural and epigenetic modifications of neuron. We examined 2D:4D in schizophrenia patients and normal controls to investigate the relationship between prenatal sex hormone exposure and genesis of schizophrenia.

Methods: The subjects were 187 schizophrenia patients(male:94, female:93), and 190 normal controls(male:95, female:95). Handedness was measured with Edinburgh Handedness Inventory. Age of onset was examined by clinical records or questioning directly to the patients. The length of digit was measured by vernier caliper. T-test, ANOVA and ANCOVA were performed to analyze the data.

Results: There were no significant differences of the 2D:4D between schizophrenia and normal controls. Also, there was no significant correlation between 2D:4D and the age of onset. Among normal controls, 2D:4D was significantly higher (more feminized) in females than in males $(\mathrm{F}=4.937, \mathrm{p}=.027)$. But, there were no significant sex differences of 2D:4D among schizophrenia patients $(\mathrm{F}=3.429, \mathrm{p}=.066)$. 